Research Article

\title{
Model Updating Method Based on Kriging Model for Structural Dynamics
}

\author{
Hong Yin $\mathbb{D}^{\mathbb{D}}$, Jingjing Ma $\left(\mathbb{D}\right.$, Kangli Dong $\mathbb{D}$, Zhenrui Peng $\mathbb{D}$, Pan Cui $\mathbb{D}^{\mathbb{D}}$, \\ and Chenghao Yang $\mathbb{D}$
}

School of Mechatronic Engineering, Lanzhou Jiaotong University, Lanzhou 730070, China

Correspondence should be addressed to Zhenrui Peng; pengzr@mail.lzjtu.cn

Received 2 January 2019; Revised 12 March 2019; Accepted 9 April 2019; Published 23 April 2019

Guest Editor: Piotr Kohut

Copyright (c) 2019 Hong Yin et al. This is an open access article distributed under the Creative Commons Attribution License, which permits unrestricted use, distribution, and reproduction in any medium, provided the original work is properly cited.

\begin{abstract}
Model updating in structural dynamics has attracted much attention in recent decades. And high computational cost is frequently encountered during model updating. Surrogate model has attracted considerable attention for saving computational cost in finite element model updating (FEMU). In this study, a model updating method using frequency response function (FRF) based on Kriging model is proposed. The optimal excitation point is selected by using modal participation criterion. Initial sample points are chosen via design of experiment (DOE), and Kriging model is built using the corresponding acceleration frequency response functions. Then, Kriging model is improved via new sample points using mean square error (MSE) criterion and is used to replace the finite element model to participate in optimization. Cuckoo algorithm is used to obtain the updating parameters, where the objective function with the minimum frequency response deviation is constructed. And the proposed method is applied to a plane truss model FEMU, and the results are compared with those by the second-order response surface model (RSM) and the radial basis function model (RBF). The analysis results showed that the proposed method has good accuracy and high computational efficiency; errors of updating parameters are less than $0.2 \%$; damage identification is with high precision. After updating, the curves of real and imaginary parts of acceleration FRF are in good agreement with the real ones.
\end{abstract}

\section{Introduction}

The accurate finite element model (FEM) is the basis of reflecting structural dynamics characteristics and guiding the optimization design of the structure. However, due to the modelling error and other uncertain factors, there is always a certain discrepancy between the FEM and the accurate model. Difference always exists between the mathematical model and experimentally measured one. Therefore, it is a general practice to update the theoretical model by using experimental measurements in order to obtain a more accurate model. Finite element model updating (FEMU) can be defined as the process of tuning a FEM to minimize the discrepancy between the measured and computed responses of the structure [1]. FEMU has attracted significant attention from the structural engineering community because of its applications in structural dynamics [2]. The current mainstream FEMU methods can be divided into two categories: FEMU based on modal parameters and FEMU based on frequency response function (FRF). The former needs modal parameter identification, which inevitably introduces the identification error, and requires that the measured degree of freedom (DOF) be consistent with the DOF of the analytical model [3]. FEMU based on FRF does not need to carry out modal analysis and mode matching, so it is suitable for structures with relatively dense modal distribution [4]. In addition, the FRF contains much information, which increases the available data and has a wider range of applications [5].

The results of structural model updating are important for engineers to accurately model similar types of structures in the future. Updated numerical models can be used in numerous engineering fields (e.g., structural vibration control, force identification, vehicle load identification of bridge systems, and structural damage detection) [6]. One of the main limitations of the FEMU in the engineering application is the computational efficiency. In theory, the higher the density of the finite element mesh, the higher the 
accuracy of the structure response prediction and the more reliable the model updating result. However, a fine finite element mesh will result in a significant increase in the computational time of the individual theoretical model response. Methods for constrained nonlinear optimization are typically used to solve the inverse problem of FEMU considering an objective function describing the discrepancies between the predicted FEM response and the measured one. Complex relationship exists between the model parameters to be identified and the objective function. The objective function may include many local minima. Gradient-based methods are commonly used for FEMU [7]. However, they might be trapped in local minima and their solution heavily depends on the starting point (i.e., initial guess of the model parameters). In order to avoid this issue, different global optimization algorithms have been used for FEMU [2]. The computational costs of the above methods are high. Surrogate model can provide a cheaper but lowerfidelity solution. The surrogate model is gaining popularity as a way of fast developed approximation for timeconsuming simulations [8].

The surrogate model does not need the complicated calculation as the FEM does. It is an approximate model constructed directly using the relationship between inputs (design parameters) and outputs (structural responses). The commonly used surrogate models include response surface method (RSM), neural network (NN), radial basis function (RBF), support vector regression (SVR), Kriging model, and so on [9-12]. Kriging model is an equivalent model based on Kriging interpolation. Unlike other surrogate models, Kriging model can not only give the prevaluation of unknown function but also get the error estimate of the prevaluation. Only a small number of samples can accurately describe the relationship between the inputs and outputs of the structure, which is widely used in the field of evaluation of machine tools [13], structural reliability analysis [14], and model updating [15]. In the field of FEMU, Zhang and Guo [16] applied Kriging theory to FEM confirmation to predict the response of the structure. Jensen et al. [17] integrated an adaptive metamodel into a FEMU using dynamic response data. The updating technique is combined with an adaptive surrogate model based on Kriging interpolation of the measure-of-fit function. Yang et al. [18] presented a new surrogate model-based FEMU taking advantage of the measured FRFs. To improve the accuracy of the surrogate model, Han et al. [19] proposed a novel formulation of gradient-enhanced surrogate model, called weighted gradient-enhanced Kriging, in combination with the adjoint method to ameliorate the curse of dimensionality for highdimensional surrogate model and design optimizations. Leifsson et al. [20] presented a method for multiobjective design optimization with variable-fidelity models and response surface surrogates, and the underlying low-fidelity model is corrected using parameterized output space mapping.

However, most methods for constructing Kriging model need to take some samples and to build a Kriging model with certain precision based on the samples and their corresponding responses. Then, the Kriging model takes place of the FEM to carry out iterative optimization. If the precision of the constructed Kriging model is too low, it may result in poor or even failed optimization results. In this case, samples must be reextracted and the Kriging model must be reconstructed. In addition, most of the methods have chosen the frequency as the response of the surrogate model. However, as an important part of model updating, FRF can provide more structural dynamic information. Unfortunately, FRF is rarely used as the response of surrogate model. Therefore, in this paper, the Kriging model with good simulation effect is applied to the model updating based on FRF data. The initial Kriging model is constructed by the parameters to be modified and the acceleration frequency response functions. In order to improve its accuracy, new sample points are added with the mean square error (MSE) criterion, and the Kriging model is updated iteratively. Then, the Kriging model which meets the accuracy requirement is applied to model updating of a truss structure. The Cuckoo algorithm with better global optimization ability is used to optimize the parameters. The numerical example verifies the effectiveness of the proposed method.

The remainder of this paper is organized as follows: Section 2 gives an overview of the Kriging model. Section 3 presents the model updating based on the Kriging model, including the MSE criterion, the objective function, and the model updating process. Section 4 provides case studies. Finally, Section 5 provides the conclusions.

\section{Kriging Model}

Kriging model is considered as the best linear unbiased estimation to the real computer model. It is a semiparametric interpolation technique which estimates the unknown information at one point according to the known information [21]. Nowadays, it has become a popular method for approximating deterministic computer model [22].

A Kriging model is a surrogate model based on a stochastic process. The model includes linear regression part and nonparametric part. And the latter can be considered as the realization of a stochastic process. For a given set of sample data (input) $\mathbf{X}=\left[x_{1}, x_{2}, \ldots, x_{n}\right]^{\mathrm{T}}$ and the observed response (output) $\mathbf{Y}=\left[y_{1}, y_{2}, \ldots, y_{n}\right]^{\mathrm{T}}$, the expression of Kriging model is

$y(\mathbf{x})=\sum_{l=1}^{p} \beta_{l} f_{l}\left(\mathbf{x}_{i}\right)+z\left(\mathbf{x}_{i}\right)=\mathbf{f}^{\mathrm{T}}\left(\mathbf{x}_{i}\right) \boldsymbol{\beta}+z\left(\mathbf{x}_{i}\right), \quad i=1,2, \ldots, n$,

where $\boldsymbol{\beta}=\left[\beta_{1}, \beta_{2}, \ldots, \beta_{p}\right]^{\mathrm{T}}$ is the coefficient vector to be estimated; $\mathbf{f}(\mathbf{x})=\left[f_{1}(x), f_{2}(x), \ldots, f_{p}(x)\right]^{\mathrm{T}}$ is the polynomial vector of the sample $\mathbf{x} ; p$ is the number of $f_{l}(\mathbf{x}) ; n$ is the number of sample points; and $z(\mathbf{x})$ represents error and is assumed to be a stochastic process that follows a normal distribution $N\left(0, \sigma^{2}\right)$ with a zero mean and standard deviation $\sigma$ generally and has nonzero covariance estimated by

$$
\operatorname{Cov}\left[z\left(\mathbf{x}_{i}\right), z\left(\mathbf{x}_{j}\right)\right]=\sigma^{2} R\left(\mathbf{x}_{i}, \mathbf{x}_{j}\right), \quad i, j=1,2, \ldots, n,
$$

where $\mathbf{x}_{i}$ and $\mathbf{x}_{j}$ are two sample points; $\sigma^{2}$ is the variance of $z(\mathbf{x})$; and $R\left(\mathbf{x}_{i}, \mathbf{x}_{j}\right)$ is the correlation function characterizing 
the correlation between any two sample points. Different $R\left(\mathbf{x}_{i}, \mathbf{x}_{j}\right)$ may induce different approximation accuracy of the constructed Kriging model. To estimate the stochastic process $z(\mathbf{x})$, assuming that the true response surface of Kriging model is continuous, any two points will tend to have the same value as the distance approaches zero and it is the same for $z(\mathbf{x})$ of two points. Thus, the correlation between $z(\mathbf{x})$ of any two sample points can be expressed as a function of their spatial distance. The most widely used Gaussian correlation model is adapted [18]:

$$
R\left(\mathbf{x}_{i}, \mathbf{x}_{j}\right)=\exp \left(-\sum_{k=1}^{m} \theta_{k}\left|x_{i}^{k}-x_{j}^{k}\right|^{2}\right),
$$

where $x_{i}^{k}$ and $x_{j}^{k}$ are the $k$ th components of the two sample points $\mathbf{x}_{i}$ and $\mathbf{x}_{j}$, respectively; $m$ denotes the number of design variables; and $\theta_{k}$ is the correlation coefficient which controls the decay rate of correlation on different dimensions. And then the matrix of correlation functions can be expressed as

$$
\mathbf{R}=\left[\begin{array}{ccc}
R\left(\mathbf{x}_{1}, \mathbf{x}_{1}\right) & \cdots & R\left(\mathbf{x}_{1}, \mathbf{x}_{n}\right) \\
\vdots & \ddots & \vdots \\
R\left(\mathbf{x}_{n}, \mathbf{x}_{1}\right) & \cdots & R\left(\mathbf{x}_{n}, \mathbf{x}_{n}\right)
\end{array}\right]
$$

When training the parameters in the Kriging model, the maximum likelihood estimation is usually used. The likelihood function of the sample point can then be expressed as

$$
L=\frac{1}{\left(2 \pi \sigma^{2}\right)^{n / 2}|\mathbf{R}|^{1 / 2}} \exp \left[-\frac{(\mathbf{Y}-\mathbf{F} \beta)^{\mathrm{T}} \mathbf{R}^{-1}(\mathbf{Y}-\mathbf{F} \boldsymbol{\beta})}{2 \sigma^{2}}\right],
$$

where $|\mathbf{R}|$ is the determinant of $\mathbf{R}$, which is a function of $\theta_{k} ; \mathbf{Y}$ is a column vector which is composed of the outputs (responses) of training samples; and $\mathbf{F}$ is a matrix of vector $\mathbf{f}(\mathbf{x})$ from each sample point. The least squares estimations of $\boldsymbol{\beta}$ and $\sigma^{2}$ can be expressed as

$$
\begin{aligned}
\widehat{\boldsymbol{\beta}} & =\left(\mathbf{F}^{\mathrm{T}} \mathbf{R}^{-1} \mathbf{F}\right)^{-1} \mathbf{F}^{\mathrm{T}} \mathbf{R}^{-1} \mathbf{Y}, \\
\widehat{\sigma}^{2} & =\frac{(\mathbf{Y}-\mathbf{F} \boldsymbol{\beta})^{\mathrm{T}} \mathbf{R}^{-1}(\mathbf{Y}-\mathbf{F} \boldsymbol{\beta})}{n} .
\end{aligned}
$$

Substituting equation (6) and equation (7) in equation (5) and ignoring the constant term, the logarithmic form of the maximum likelihood function can be expressed as

$$
\ln (L) \approx-\frac{n}{2} \ln \left(\widehat{\sigma}^{2}\right)-\frac{1}{2} \ln |\mathbf{R}| .
$$

Both $\widehat{\sigma}$ and $\mathbf{R}$ are the functions of $\theta_{k}$. And they can be obtained from the maximum likelihood estimation of the response. So, by solving the maximum value of the above expression, the value of $\theta_{k}$ can be determined.

Stochastic distribution $z(\mathbf{x})$ (in equation (1)) is sufficient to indicate the changing trend of the function response, so the regression part $\mathbf{f}^{\mathrm{T}}(\mathbf{x})$ is regarded as a constant term. Then, the unique unknown parameter in the Kriging model is $\theta_{k}$, which can be obtained through optimization algorithm.

After the Kriging model is constructed, the next step is to predict the response value of the point to be measured. For any point $\mathbf{x}_{0}$ to be measured, its response value $\hat{y}\left(\mathbf{x}_{0}\right)$ can be expressed as

$$
\hat{y}\left(\mathbf{x}_{0}\right)=\mathbf{f}^{\mathrm{T}}\left(\mathbf{x}_{0}\right) \widehat{\boldsymbol{\beta}}+\mathbf{r}^{\mathrm{T}}\left(\mathbf{x}_{0}\right) \mathbf{R}^{-1}(\mathbf{Y}-\mathbf{F} \widehat{\boldsymbol{\beta}}) .
$$

The prediction accuracy can be evaluated by estimating MSE of the predicted value, which can be estimated as

$$
\widehat{\boldsymbol{s}}^{2}(\mathbf{x})=\sigma^{2}\left[1-\left\{\mathbf{f}^{\mathrm{T}}(\mathbf{x}), \mathbf{r}^{\mathrm{T}}(\mathbf{x})\right\}\left[\begin{array}{ll}
0 & \mathbf{F}^{\mathrm{T}} \\
\mathbf{F} & \mathbf{R}
\end{array}\right]^{-1}\left\{\begin{array}{l}
\mathbf{f}(\mathbf{x}) \\
\mathbf{r}(\mathbf{x})
\end{array}\right\}\right],
$$

where $\mathbf{r}^{\mathrm{T}}\left(\mathbf{x}_{0}\right)$ is the row vector of correlation function between each sample point and the point $\mathbf{x}_{0}$ to be measured:

$$
\mathbf{r}^{\mathrm{T}}\left(\mathbf{x}_{0}\right)=\left[R\left(\mathbf{x}_{0}, \mathbf{x}_{1}\right), R\left(\mathbf{x}_{0}, \mathbf{x}_{2}\right), \ldots, R\left(\mathbf{x}_{0}, \mathbf{x}_{n}\right)\right] .
$$

\section{Kriging-Based Model Updating Method}

3.1. Improvement of Kriging Model. The accuracy of Kriging model directly affects the results of model updating. Constructing a high-precision Kriging model requires a large number of sample points. However, the number of sample points is the key to restrict the computational efficiency of Kriging model. The prediction of each new point needs to compute the correlation between the new sample point and all sample points. Therefore, in order to ensure the computational efficiency, the number of sample points should not be too large. But small number of sample points may lead to low accuracy of the model. In order to make Kriging model have a good compromise between computational efficiency and accuracy, it is necessary to add new sample points in effective area according to certain rules (infill criteria).

At present, the infill criteria mainly include improved expectation criterion (EI criterion), improved probability criterion (PI criterion), confidence lower bound criterion (LCB criterion), and mean square error criterion (MSE criterion) [12]. In this paper, the MSE criterion is selected to add new sample points for updating the Kriging model. The estimation of root mean square error (RMSE) provided by the Kriging model is used to guide the addition of new sample points, thereby improving the global accuracy of the model. Relative to the optimization of model updating, the optimization here is referred as suboptimization. In short, it is to use a reasonable optimization algorithm to solve the following optimization problem so as to get a new sample point:

$$
\begin{aligned}
& \operatorname{Max} \operatorname{MSE}(\hat{y}(\mathbf{x})), \quad \mathbf{x}_{1} \leq \mathbf{x} \leq \mathbf{x}_{\mathrm{u}}, \\
& \operatorname{MSE}(\hat{y}(\mathbf{x}))=s^{2}(\mathbf{x})=\sigma^{2}\left(1-\mathbf{r}^{\mathrm{T}} \mathbf{R}^{-1} \mathbf{r}+\frac{\left(1-\mathbf{F}^{\mathrm{T}} \mathbf{R}^{-1} \mathbf{r}\right)^{2}}{\mathbf{F}^{\mathrm{T}} \mathbf{R}^{-1} \mathbf{F}}\right),
\end{aligned}
$$

where $\mathbf{x}_{1}$ is the lower bound of design parameters; $\mathbf{x}_{\mathrm{u}}$ is the upper bound of design parameters; $\hat{y}(\mathbf{x})$ is the predicted response of the Kriging model at the sample point; $s^{2}(\mathbf{x})$ denotes the MSE estimation of the Kriging model; $\sigma$ is the variance of the stochastic process; $\mathbf{R}$ is the matrix of correlation functions between sample points; $\mathbf{R}^{-1}$ is the inverse 
of $\mathbf{R} ; \mathbf{r}$ is a correlation vector, consisting of correlation functions between unknown points and all known sample points; $\mathbf{F}$ is a matrix of polynomial vector for each sample point; and $\mathbf{F}^{\mathrm{T}}$ is the transpose of $\mathbf{F}$.

It is necessary to choose a suitable convergence criterion to terminate the surrogate infill process. Here, the maximum number of new samples is taken as the termination criterion. The solution for the location of the maximum RMSE is finished by Cuckoo algorithm.

The main steps of updating the Kriging model can be described as follows:

(1) Construct the initial Kriging model according to the theory in Section 2.

(2) Compute the maximum RMSE value of the current Kriging model by the Cuckoo algorithm and add it into the set of sample points.

(3) Run the finite element analysis program to compute the responses of the new sample points and update the initial Kriging model until the set termination condition is reached.

The RMSE distribution of one-dimensional Schwefel function is shown in Figure 1. As shown in Figure 1(a), the sample point corresponding to the maximum RMSE is around 0.8 . Figure 1(b) is obtained after adding this sample point; it can be seen that the precision of the surrogate model is greatly improved. Therefore, it is an effective method to update the Kriging model by using the optimization algorithm step by step to select the point with the largest RMSE as the new sample point.

3.2. Objective Function. Essentially, model updating is an optimization problem. Minimizing the objective function, the modified values of design parameters are obtained by solving the optimization problem. In this paper, the objective function is established based on the differences of the acceleration frequency response function between the experimental model and Kriging model.

In the frequency domain, the general mathematical representation of $n$ DOFs system is expressed as

$$
\left(-\omega^{2} \mathbf{M}+i \omega \mathbf{C}+\mathbf{K}\right) \mathbf{X}(\omega)=\mathbf{F}(\omega),
$$

where $\mathbf{M}, \mathbf{K}$, and $\mathbf{C}$ are $n \times n$ matrices of mass, stiffness, and damping, respectively. $\mathbf{X}(\omega)$ is $n \times 1$ displacement response vector subjected to the applied force vector of $\mathbf{F}(\omega)$. $\omega$ is the excitation frequency. The displacement vector $\mathbf{X}(\omega)$ can be achieved by

$$
\mathbf{X}(\omega)=\mathbf{H}(\omega) \mathbf{F}(\omega)
$$

where $\mathbf{H}(\omega)$ is a $n \times n$ FRF matrix defined as

$$
\mathbf{H}(\omega)=\left[\mathbf{M}-\frac{i \mathbf{C}}{\omega}-\frac{\mathbf{K}}{\omega^{2}}\right]^{-1} .
$$

The objective function here is defined as

$$
\mathrm{Obj}=\sum_{i=1}^{\max F}\left|\widehat{y}\left(\omega_{i}\right)-\log _{10} H\left(\omega_{i}\right)\right|,
$$

where $\hat{y}\left(\omega_{i}\right)$ represents the prediction response of the updated Kriging model at $\omega_{i} ; \max F$ denotes the maximum observation frequency; and $H\left(\omega_{i}\right)$ represents the response of the structure at $\omega_{i}$. The peak value of the FRF is usually large at some frequency points, which will affect the prediction accuracy of the Kriging model, so the logarithmic (log $(\mathrm{FRF}))$ is taken as the response.

Many optimization algorithms can be used to solve equation (17). The Cuckoo algorithm [23] has strong global search ability due to its unique Lévy performance. Moreover, it has the advantages of less parameters, simple operation, easy implementation, and good optimization performance. Therefore, the Cuckoo algorithm is used to solve the parameters to be modified.

3.3. Process of Model Updating. As mentioned above, when the Kriging model is introduced into the structural FEMU, the Kriging model replaces the FEM to participate in optimization, and thus FRF model updating is fulfilled.

The main steps of model updating method based on Kriging model can be summarized as follows:

Step 1: select the excitation point and measurement point and determinate the parameters to be modified.

Step 2: generate initial sample points of the modified parameters by design of experiment (DOE).

Step 3: run the finite element analysis program to compute the response of the sample points and construct the initial Kriging model.

Step 4: find the point with the maximum RMSE of the current Kriging model by the Cuckoo algorithm and add it into the set of sample points.

Step 5: compute the responses of the new sample points and update the initial Kriging model.

Step 6: check whether the maximum number of new samples is satisfied. If it is, go to Step 7. Otherwise, go back to Step 4 and continue adding new points.

Step 7: take equation (17) as the target; the updated Kriging model is used to replace the FEM to participate in optimization, and the modified values of parameters are obtained by the Cuckoo algorithm.

The flow chart of model updating is shown in Figure 2.

\section{Numerical Example}

A plane truss structure (Figure 3) is taken as an example to verify the model updating method. The truss has 14 nodes and 25 DOFs. The elasticity modulus of each bar element is $210 \mathrm{GPa}$ and the density is $7850 \mathrm{~kg} \cdot \mathrm{m}^{-3}$. Select the first fourth modes as the modes of interest. 


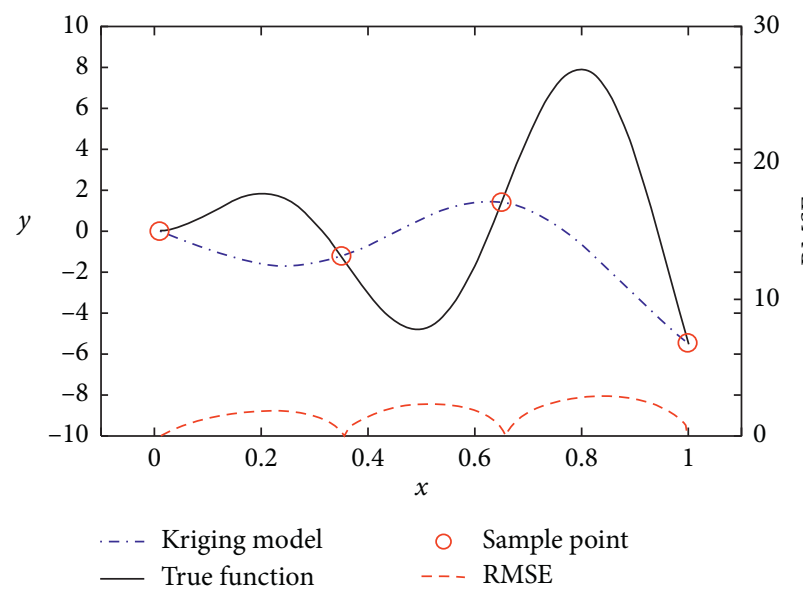

(a)

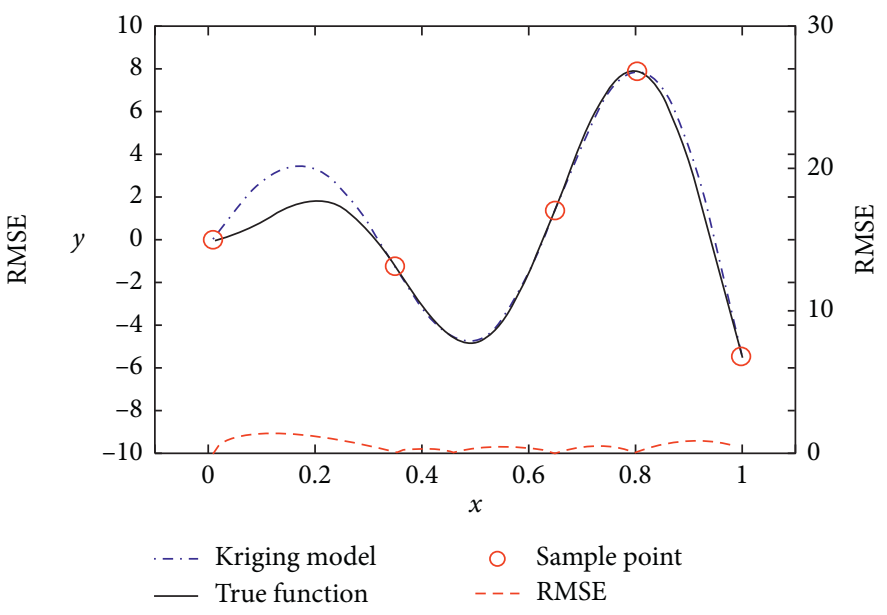

(b)

FIGURE 1: RMSE distribution of Schwefel function. (a) Before adding point. (b) After adding point.

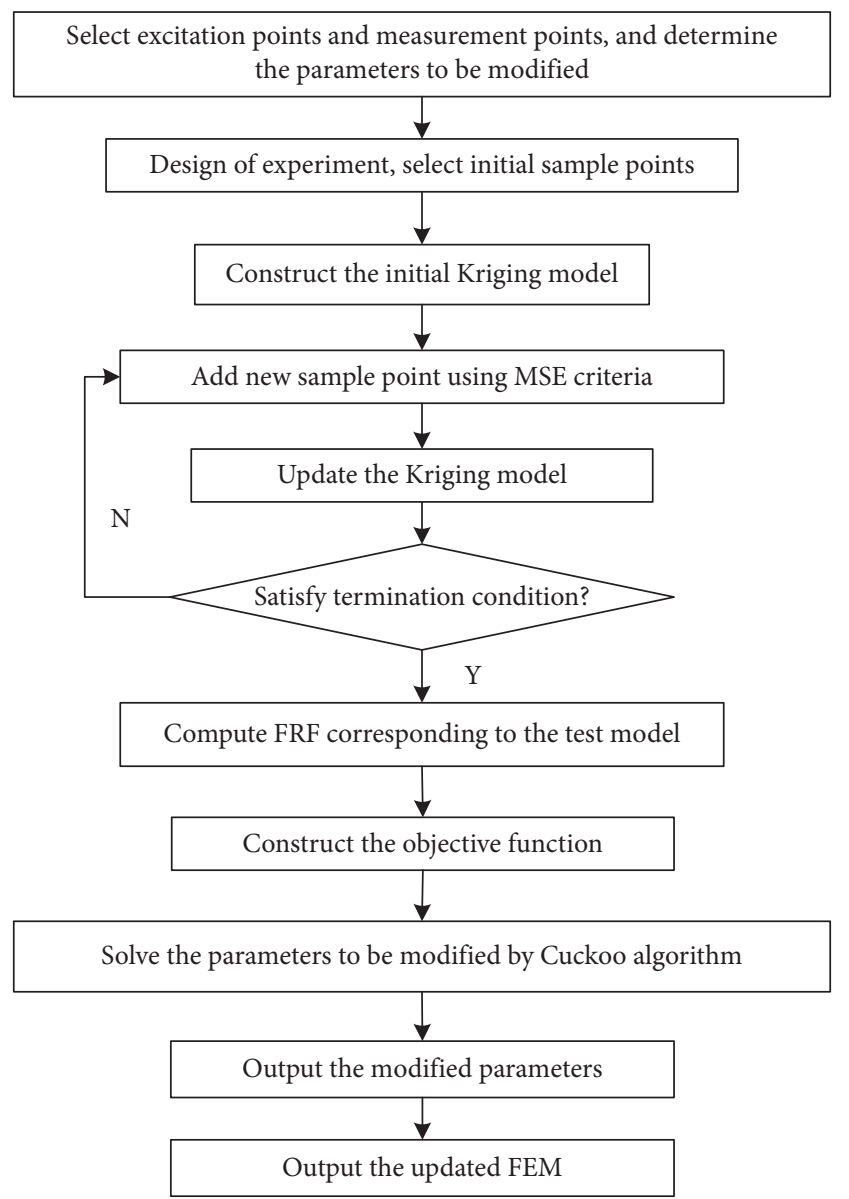

FIGURE 2: Flow chart of model updating.

4.1. Selection of Excitation Points and Measurement Points. Modal participation criterion is used for selecting excitation points. Modal participation [24] is applied to evaluate the contribution of each DOF to the excitation modes of the structure. Modal participation can be expressed as

$$
W_{q i}=\sum_{p=1}^{N_{0}}\left|A_{p q i}\right|, \quad q=1,2, \ldots, N_{0}, i=1,2, \ldots, r,
$$

where subscripts $p$ and $q$ denote the output DOFs and input DOFs, respectively; $r$ represents number of modes; $N_{0}$ represents the number of DOFs; and $A_{p q i}$ represents the residue.

Assuming that the structure is proportional damping, the FRF can be expressed as

$$
H_{\mathrm{pq}}(\omega)=\sum_{i=1}^{m} \frac{\phi_{p i} \phi_{q i}}{\omega_{i}^{2}-\omega^{2}+2 i \xi_{i} \omega_{i} \omega},
$$

where $\phi_{p i}$ and $\phi_{q i}$ represent the $(i, p)$ th and $(i, q)$ th elements the modal matrix, respectively; $\omega_{i}$ denotes the undamped natural frequency at the $i$ th order mode; and $\xi_{i}$ represents the damped coefficient at the $i$ th order mode. $A_{p q i}$ in equation (7) can be expressed as

$$
A_{p q i}=\phi_{p i} \phi_{q i} \text {. }
$$

The contribution of the qth DOF to the excitation of all modes of interest can be expressed as

$$
W_{q}=\sum_{i=1}^{r} W_{q i}, \quad q=1,2, \ldots, N_{0} .
$$

In single-input and single-output modal response test, structural DOF with maximum $W_{q}$ can be selected as the optimal excitation point. $W_{\mathrm{qi}}$ and $W_{q}$ of each DOF of the structure are shown in Figure 4. The maximum $W_{q}$ is at the 20th DOF in the $Y$-direction of node 11. So, the 20th DOF is chosen as the best excitation point in the test. The DOFs in the $Y$-direction make the most important contribution to the first four modes of excitation. This is consistent with the characteristics of the plane truss structure.

There are also many methods for selecting measurement points, but most of them are multipoint selection. According to the characteristics of the model, a DOF in the $Y$-direction should be selected as the sensor measurement point. The 


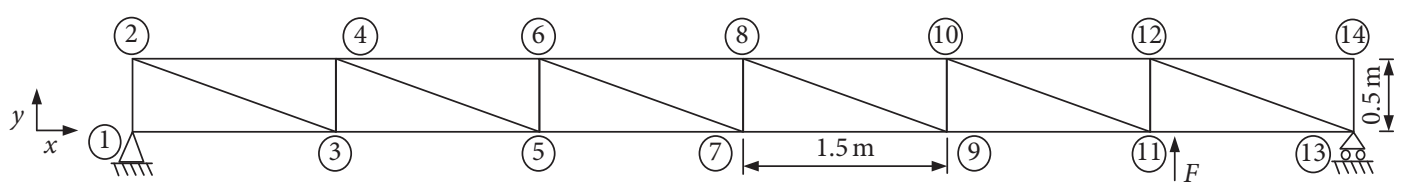

Figure 3: Plane truss model.

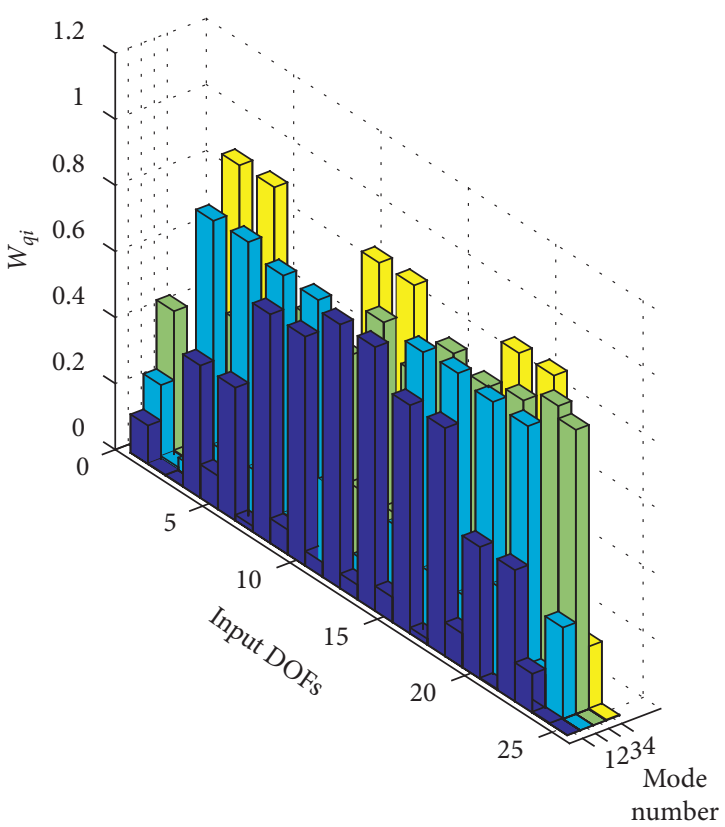

(a)

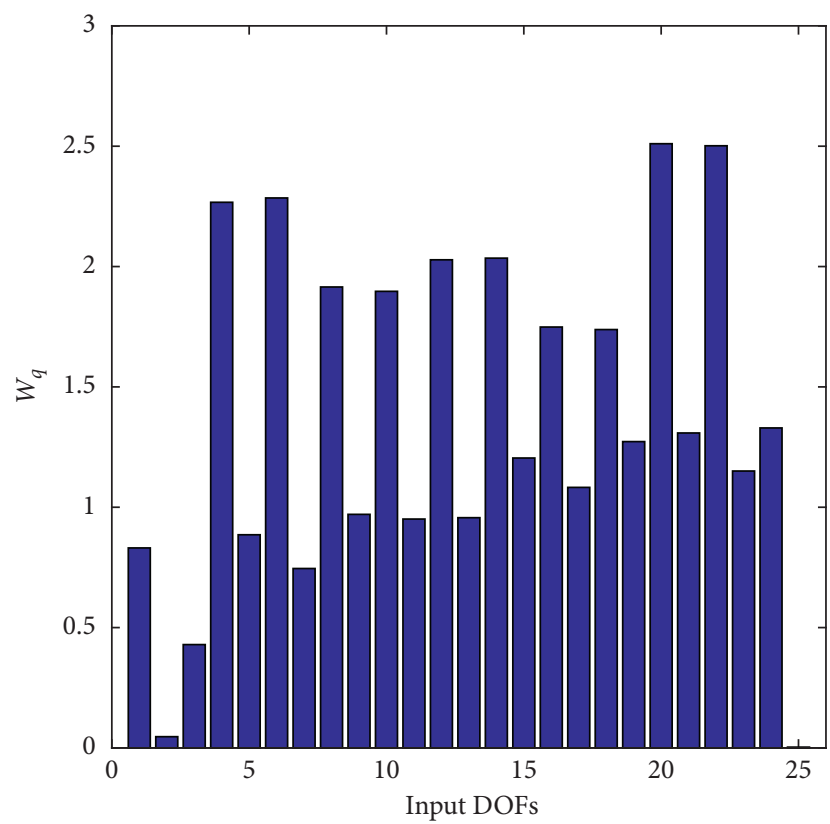

(b)

Figure 4: Value of modal participation. (a) $W_{\mathrm{qi}}$. (b) $W_{q}$.

fourth DOF in the $Y$-direction at the 3rd node is chosen as the measurement point.

4.2. Test Design and Initial Sample Selection. In this paper, the FEM shown in Figure 3 is used as the test model, and the corresponding FEM is obtained by deviating from the values of the parameters to be modified in the test model. Considering that the test data will be affected by noise in the actual data measurement, $5 \%$ random noise is added to the response of the test model. The elasticity modulus and material density are chosen as parameters to be modified and their values are deviated. The elasticity modulus is increased by $10 \%$. The material density is reduced by $10 \%$. The FEM parameters are shown in Table 1.

Before constructing the surrogate model, selecting the sample points is the first step. For the global optimization problem, a better method is to select a set of sample points through DOE. The method proposed in this paper has no strict requirements for the number of initial sample points, which is different from the traditional Kriging model. Considering the efficiency of adding new sample points, the number of initial samples should not be too small. In other words, the optimization efficiency of the improved Kriging model based on our method is not obviously dependent on the number of initial sample points. Here, the Latin
TABle 1: Parameters of test model and FEM.

\begin{tabular}{lccc}
\hline Parameters & $\begin{array}{c}\text { Test } \\
\text { value }\end{array}$ & $\begin{array}{c}\text { Initial } \\
\text { error }(\%)\end{array}$ & $\begin{array}{c}\text { FEM } \\
\text { value }\end{array}$ \\
\hline Elasticity modulus $(\mathrm{GPa})$ & 210 & 10 & 231 \\
Material density $\left(\mathrm{kg} \cdot \mathrm{m}^{-3}\right)$ & 7850 & -10 & 7065 \\
\hline
\end{tabular}

hypercube sampling (LHS) is used to sample the two parameters (elasticity modulus and material density). Finally, 40 samples are extracted.

4.3. Construction and Verification of Kriging Model. On the basis of the 40 extracted samples and their corresponding acceleration FRFs, the initial Kriging model is constructed. Then, the new samples are added according to the MSE criterion introduced in Section 3, and the maximum number of additional sample points is 40. Then, the improved Kriging model is built. The FRFs at the fourth DOF of the FEM are predicted by using Kriging model and the improved Kriging model, respectively. The results are shown in Figure 5 (RMSE1 denotes RMSE of Kriging model; RMSE2 denotes RMSE of the improved Kriging model). Both the Kriging model and the improved Kriging model have good prediction accuracy. However, the improved Kriging model performs better in predicting the peak value of the curve, and 


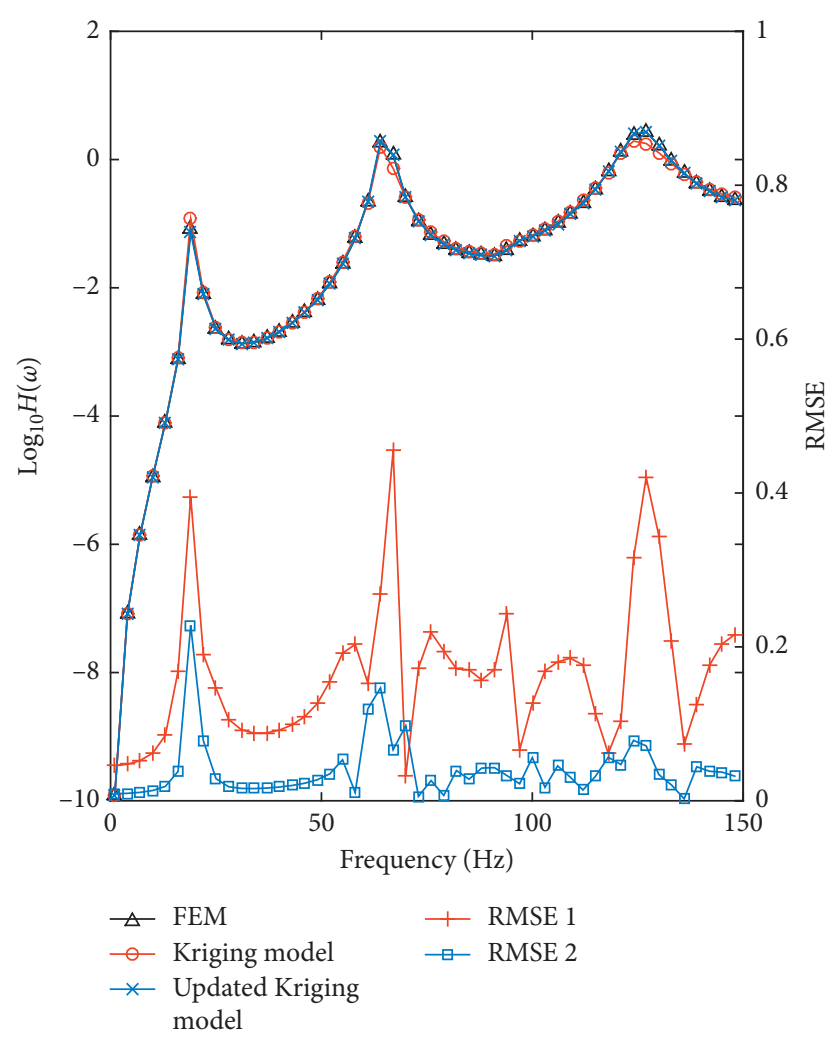

Figure 5: Log(FRF) of FEM.

the RMSE value of the improved Kriging model is smaller than that of the Kriging model.

The fitting response surface and RMSE surface obtained by updating the Kriging model at the 50th frequency point are shown in Figure 6. The overlap between the sample response and the predicted response from the improved Kriging model is good, and the maximum RMSE is less than 0.05 . The improved Kriging model has good approximation accuracy to the FEM.

4.4. Model Updating. The improved Kriging model mentioned above is used to substitute the FEM to optimize iteratively. Assume that the test parameters are within the interval of finite element parameter values. The Cuckoo algorithm is used to find the optimum iteratively. The number of nests is 40 , and the maximum number of iterations is 100. In order to prove the stability of the algorithm, the iterative convergence curve is shown in Figure 7 when the algorithm runs 100 times. The Cuckoo algorithm is stable and converges before the number of iterations reaches 60 . The difference between the optimal value and the worst value is also very small.

In order to compare with the optimization effect of the updated Kriging model, the second-order RSM and the RBF are constructed based on the same sample points. The average values and average errors of the modified parameters are shown in Table 2. Except for the second-order RSM, all the other three methods obtain good results, but the parameter values by the improved Kriging model are more accurate, and the average error is the smallest.

All the algorithms are coded in Matlab 2014b. The operating system is Windows 10. Simulation hardware is a PC with $3.50 \mathrm{GHz}$ Intel Pentium (R) G4560 and $12.00 \mathrm{~GB}$ memory, and all the algorithms are repeated 5 times. The total computation time of model updating using these surrogate models is counted, and the average time is shown in Table 3. The RSM takes the least time. The RBF needs the longest time. And the Kriging model needs longer time than RSM, but its computational efficiency is much higher than RBF.

In summary, the Kriging model has the best accuracy and needs less time. The accuracy of RSM is too poor. The computation time of RBF is too long. RSM and RBF cannot be compromised between accuracy and time. This shows that the proposed method can improve the computational efficiency while satisfying the accuracy and has little dependence on the number of sample points. It is noted that there are some errors between the Kriging model and the improved Kriging model, which are not caused by the insufficient optimization ability of the optimization algorithm, but by the prediction error of the Kriging model itself. So, only if the Kriging model is accurate enough, it can be used to modify the structure model to reduce the computational cost and get the accurate updating results.

The FRFs predicted by the Kriging model, the updated Kriging model, RBF, and RSM are shown in Figure 8. Comparison of updated Kriging model with Kriging model, RSM, and RBF is shown in Figures $8(\mathrm{a})$ and $8(\mathrm{~b})$, respectively (RMSE1 denotes RMSE of Kriging model; RMSE2 denotes RMSE of the updated Kriging model; RMSE3 denotes RMSE of RSM; RMSE4 denotes RMSE of $\mathrm{RBF}$ ). The prediction value of the updated Kriging model is more accurate than others. The form of FRFs of the test model and the FEM does not change, and the peak values are very close, only causing movement along the frequency axis.

The FEM is updated with the average modified values obtained from the updated Kriging model. By comparing the real and imaginary FRF curves before and after modification at the measurement points, the updating effect of the proposed method is further verified. The comparison curve between real and imaginary parts of FRF before and after updating is shown in Figure 9. The FRFs of the updated FEM (whether real or imaginary part), can coincide well with the FRFs of the test model.

4.5. Damage Identification. In order to further verify the proposed method, the above mentioned truss structure elements $8,12,15,17,21$, and 25 are assumed to be damage elements. Their stiffness of the elements is reduced by $10 \%$, $10 \%, 20 \%, 20 \%, 30 \%$, and $30 \%$, respectively. The elasticity modulus of each damaged unit is identified. The number of samples is set to 500. The maximum number of new additional sample points is 100 . The maximum number of iterations of the Cuckoo algorithm is 300 . The damage identification errors by the Kriging model, the updated Kriging model, the second-order RSM, and RBF under 


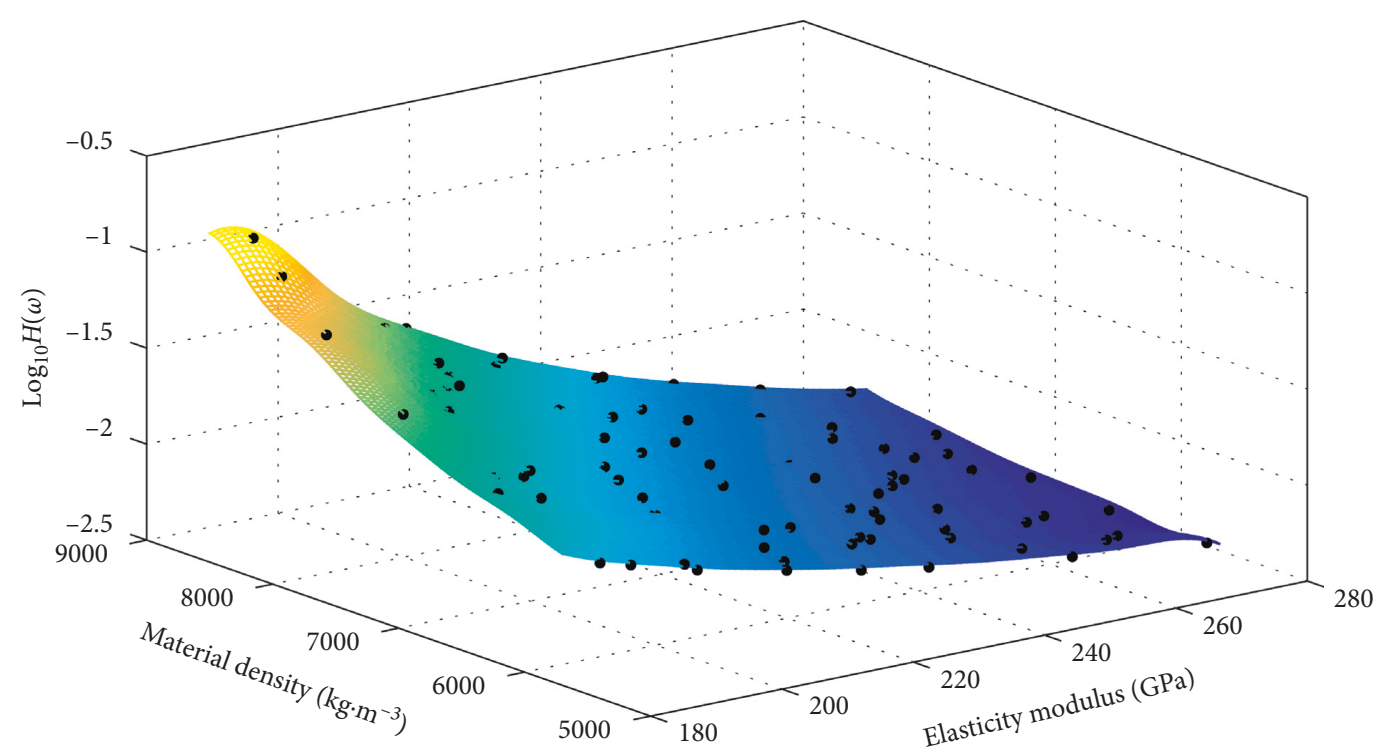

(a)

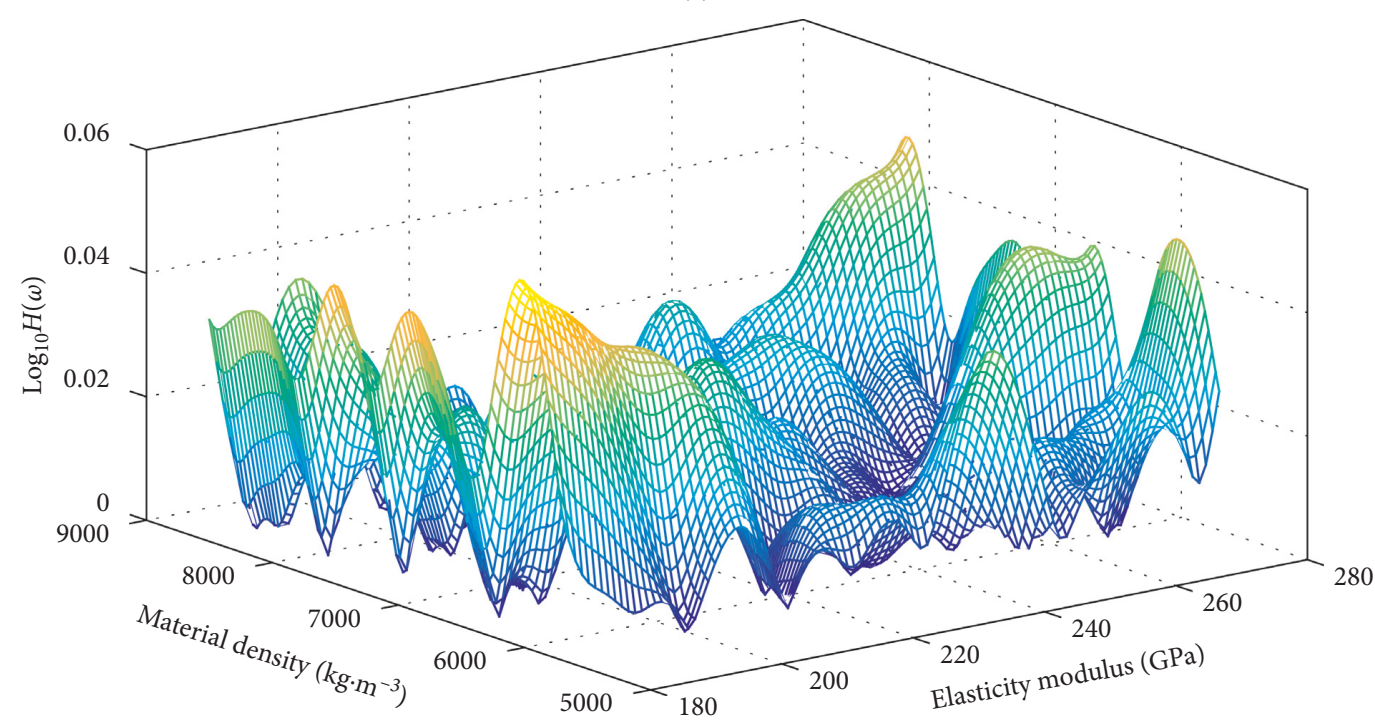

(b)

FIGURE 6: Prediction values of the improved Kriging model. (a) Response values. (b) RMSE values.

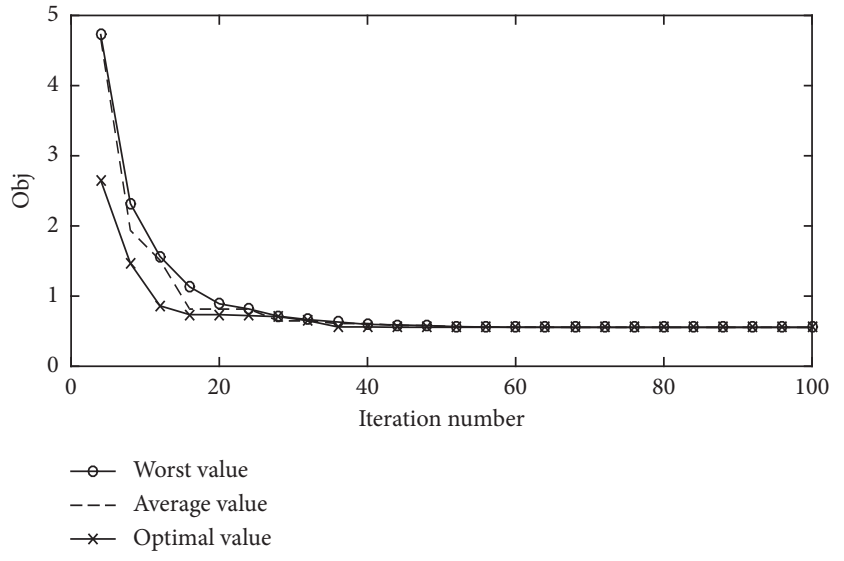

FIgURE 7: Iterative curve. different measurement points are shown in Table 4. The effects of different measurement points on damage identification accuracy are different. When sensors are evenly arranged at all $Y$-direction DOFs, 4 surrogate models have better identification accuracy. The identification error of the updated Kriging model is less than $0.5 \%$. For a single measurement point, except for $14-Y$ measurement point, the identification accuracy of the four methods for unit 12 at other measurement points is poor; at 5- $Y$ measurement point, the surrogate models have larger identification error. The identification accuracy of the updated Kriging model is better than that of the other surrogate models on the whole. For the identification of elasticity modulus of the truss damage elements, the computation time of the Kriging model is about twice that of RBF and 0.04 times that of RSM. 
TABle 2: Parameters and errors of model updating.

\begin{tabular}{lccc}
\hline & & Elasticity modulus $(\mathrm{GPa})^{\text {Material density }\left(\mathrm{kg} \cdot \mathrm{m}^{-3}\right)}$ \\
\hline & Test & 210.000 & 7850.000 \\
Values by different methods & $\mathrm{K}$ & 211.273 & 7891.508 \\
& $\mathrm{U}-\mathrm{K}$ & 210.005 & 7863.081 \\
& $\mathrm{RBF}$ & 211.285 & 7903.145 \\
& $\mathrm{RSM}$ & 210.945 & 8052.001 \\
\hline & $\mathrm{K}$ & 0.606 & 0.529 \\
Average errors by different methods (\%) & $\mathrm{U}-\mathrm{K}$ & 0.002 & 0.167 \\
& $\mathrm{RBF}$ & 0.612 & 0.677 \\
\hline
\end{tabular}

Note. K represents the Kriging model; U-K represents the updated Kriging model.

TABle 3: Computation time.

\begin{tabular}{lccc}
\hline Surrogate model & Kriging & RBF & RSM \\
\hline Average time (s) & 13.949 & 695.802 & 3.837 \\
\hline
\end{tabular}

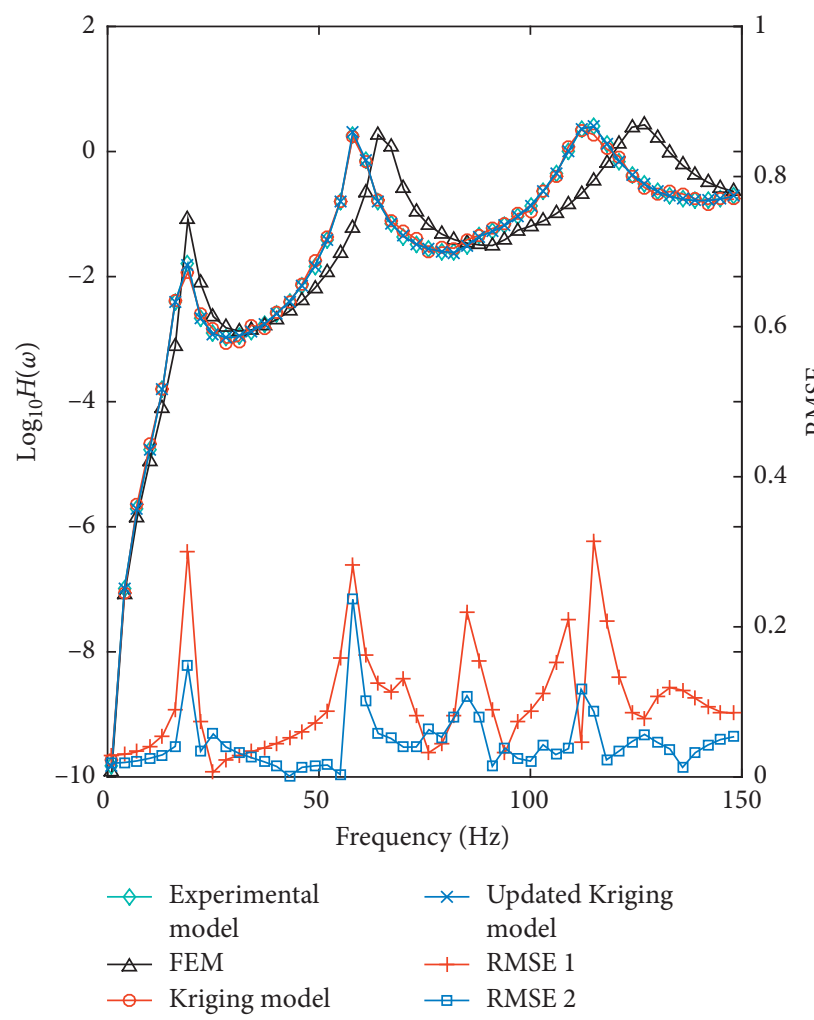

(a)

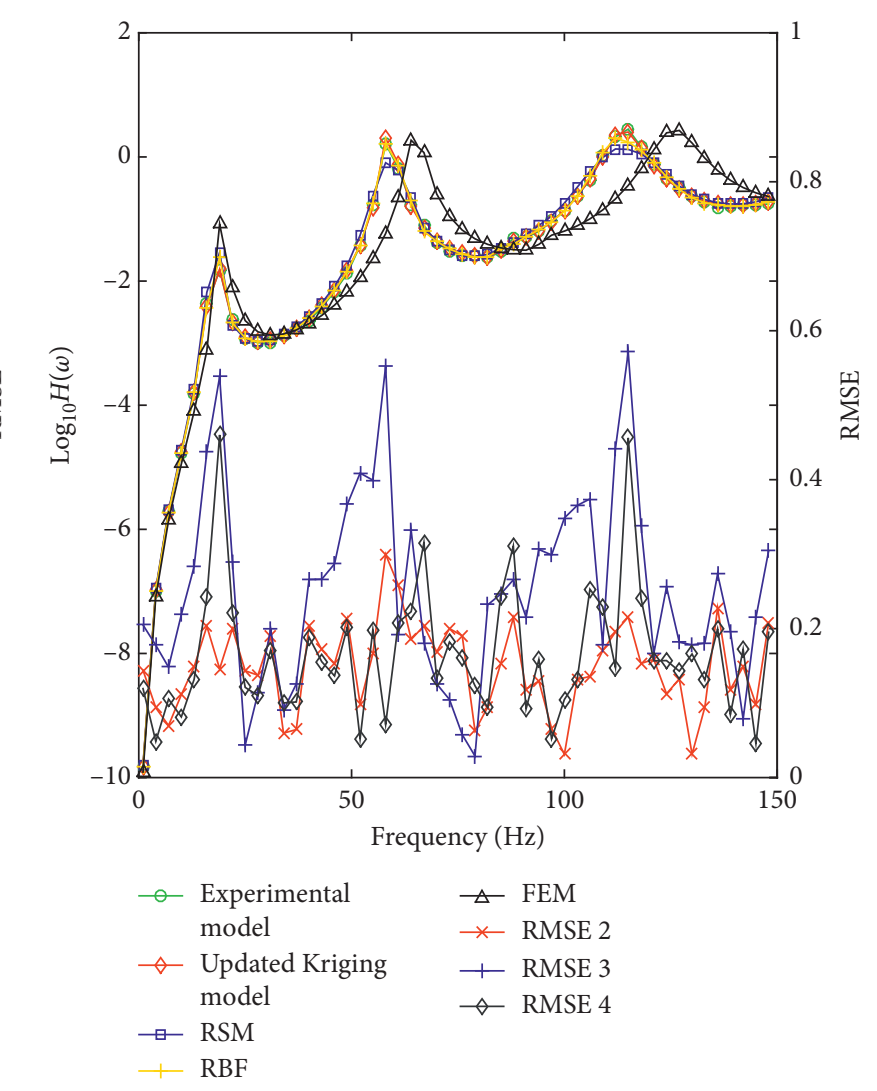

(b)

Figure 8: $\log (\mathrm{FRF})$ of experimental model. (a) Comparison of the updated Kriging model with the Kriging model. (b) Comparison of the updated Kriging model with RBF and RSM.

FEMU based on Kriging model can make a good compromise between computational accuracy and time. The accuracy of damage identification is not only affected by the surrogate models but also affected by the selection of measurement points.

\section{Conclusions}

In this paper, the updated Kriging model is used to modify the parameters and to identify the damage of the structure. The conclusions are as follows: 


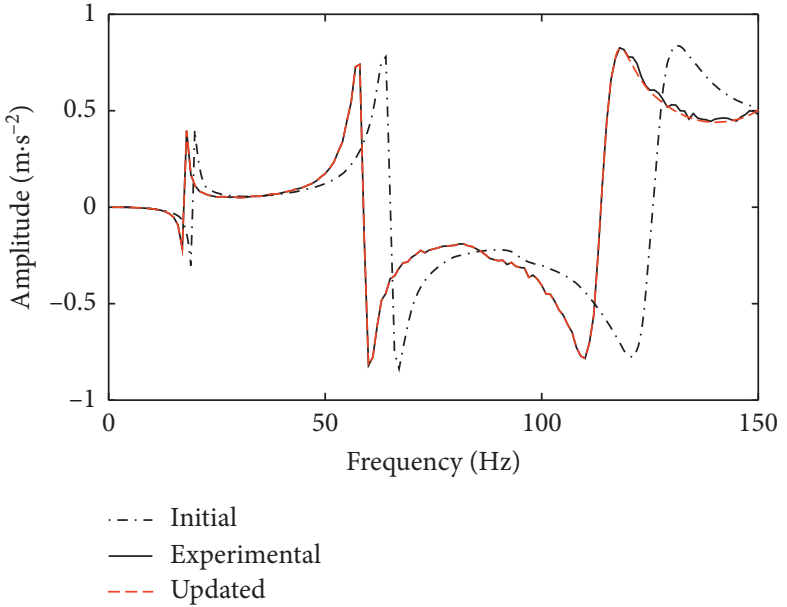

(a)

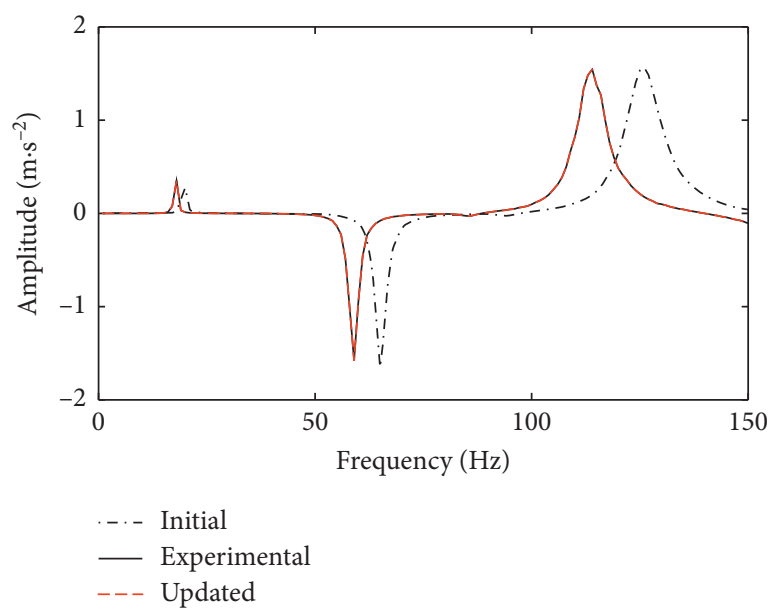

(b)

FIgURE 9: FRFs before and after being updated. (a) Real part. (b) Imaginary part.

TABLE 4: Identification error of elasticity modulus of each damage unit.

\begin{tabular}{|c|c|c|c|c|c|c|c|}
\hline \multirow{2}{*}{ Measurement location } & \multirow{2}{*}{ Method } & \multicolumn{6}{|c|}{ Identification errors of damage units (\%) } \\
\hline & & 8 & 12 & 15 & 17 & 21 & 25 \\
\hline \multirow{4}{*}{$3-Y$} & $\mathrm{~K}$ & 1.594 & 5.125 & 6.225 & 0.504 & 0.098 & 0.946 \\
\hline & $\mathrm{U}-\mathrm{K}$ & 1.315 & 3.812 & 4.334 & 0.490 & 0.099 & 0.622 \\
\hline & $\mathrm{RBF}$ & 0.487 & 2.339 & 1.750 & 0.577 & 0.006 & 0.234 \\
\hline & RSM & 1.064 & 8.371 & 6.323 & 0.229 & 0.342 & 1.064 \\
\hline \multirow{4}{*}{$5-Y$} & $\mathrm{~K}$ & 0.722 & 8.359 & 8.830 & 1.372 & 0.492 & 1.815 \\
\hline & $\mathrm{U}-\mathrm{K}$ & 0.431 & 6.488 & 6.261 & 1.253 & 0.340 & 1.406 \\
\hline & $\mathrm{RBF}$ & 0.090 & 21.078 & 20.502 & 11.459 & 1.653 & 5.297 \\
\hline & RSM & 3.882 & 11.270 & 5.399 & 6.676 & 0.588 & 1.499 \\
\hline \multirow{4}{*}{$7-Y$} & $\mathrm{~K}$ & 0.890 & 7.373 & 0.768 & 6.824 & 0.557 & 0.280 \\
\hline & U-K & 0.897 & 3.470 & 0.054 & 2.850 & 0.108 & 0.017 \\
\hline & $\mathrm{RBF}$ & 3.718 & 6.606 & 1.231 & 8.987 & 0.055 & 0.904 \\
\hline & RSM & 4.663 & 1.201 & 0.324 & 2.165 & 0.202 & 0.337 \\
\hline \multirow{4}{*}{$9-Y$} & $\mathrm{~K}$ & 0.982 & 2.746 & 0.028 & 1.506 & 0.332 & 0.073 \\
\hline & U-K & 0.685 & 2.160 & 0.030 & 1.126 & 0.183 & 0.025 \\
\hline & $\mathrm{RBF}$ & 3.469 & 1.720 & 0.096 & 1.606 & 0.081 & 0.161 \\
\hline & RSM & 4.522 & 1.879 & 0.627 & 0.626 & 0.492 & 0.743 \\
\hline \multirow{4}{*}{$11-Y$} & $\mathrm{~K}$ & 0.554 & 1.918 & 0.021 & 1.276 & 0.435 & 0.304 \\
\hline & U-K & 0.552 & 0.916 & 0.031 & 0.327 & 0.087 & 0.132 \\
\hline & $\mathrm{RBF}$ & 2.088 & 2.970 & 0.275 & 2.269 & 0.093 & 0.277 \\
\hline & RSM & 5.810 & 2.018 & 0.578 & 1.424 & 0.625 & 0.862 \\
\hline \multirow{4}{*}{$14-Y$} & $\mathrm{~K}$ & 0.226 & 0.648 & 0.084 & 0.271 & 0.546 & 0.260 \\
\hline & U-K & 0.167 & 0.783 & 0.084 & 0.268 & 0.125 & 0.075 \\
\hline & $\mathrm{RBF}$ & 1.587 & 1.197 & 0.830 & 2.244 & 0.021 & 0.574 \\
\hline & RSM & 4.519 & 1.752 & 0.639 & 0.631 & 0.478 & 0.634 \\
\hline \multirow{4}{*}{ All measurement points of $Y$-direction } & $\mathrm{K}$ & 0.170 & 0.020 & 0.232 & 0.676 & 0.287 & 0.189 \\
\hline & U-K & 0.197 & 0.010 & 0.010 & 0.423 & 0.140 & 0.163 \\
\hline & $\mathrm{RBF}$ & 0.244 & 0.486 & 0.468 & 0.155 & 0.301 & 0.289 \\
\hline & RSM & 1.284 & 1.176 & 0.184 & 0.188 & 0.319 & 0.586 \\
\hline
\end{tabular}

Note. $\mathrm{K}$ represents the Kriging model; U-K represents the updated Kriging model; 3- $Y$ represents the measurement points arranged in the $Y$ direction at the 3rd node.

(1) For modal response test, the selection of excitation point and measurement point is very important. For single-input and single-output test, modal participation criterion can be used to select the excitation point, and the response point can be selected according to the structural characteristics. 
(2) The updated Kriging model obtained by using the MSE criterion has better accuracy than the Kriging model, and the accuracy of the model is more stable.

(3) Compared with the second-order RSM and RBF, the updated Kriging model can improve the computational efficiency while satisfying the computational accuracy and has less dependence on the number of sample points.

(4) The parameter errors of the updated model are less than $0.2 \%$. When there are enough measurement points, the parameter errors of damage identification are less than $0.5 \%$.

(5) When the optimization algorithm has enough optimization performance, the parameter modification errors often depend on the accuracy of the surrogate models, which are independent of the optimization algorithm, and when the surrogate models are accurate enough, the parameter modification errors will tend to zero.

Further research is needed on the selection of multiple measurement points for model updating. In the following research, modal response test will be carried out.

\section{Data Availability}

The data used to support the findings of this study are available from the corresponding author upon request.

\section{Conflicts of Interest}

The authors declare no conflicts of interest.

\section{Acknowledgments}

This research was funded by the Natural Science Foundation of China (no. 51768035), Collaborative Innovation Team Project of Universities in Gansu Province (no. 2018C-12), Talent Project of Lanzhou City (no. 2017-RC-66), and Foundation of a Hundred Youth Talents Training Program of Lanzhou Jiaotong University.

\section{References}

[1] M. I. Friswell and J. E. Mottershead, Finite Element Model Updating in Structural Dynamics, Kluwer Academic Publishers, Dordrecht, Netherlands, 1995.

[2] R. Astroza, L. T. Nguyen, and T. Nestorović, "Finite element model updating using simulated annealing hybridized with unscented Kalman filter," Computers \& Structures, vol. 177, pp. 176-191, 2016.

[3] J. W. Zhan, M. Li, Y. Lu et al., "Dynamic evaluation method for damage of simply-supported beams based on model updating theory and FRF signature assurance criterion," China Civil Engineer Ring Journal, vol. 8, pp. 49-58, 2017.

[4] H. H. Khodaparast, J. E. Mottershead, and K. J. Badcock, "Interval model updating with irreducible uncertainty using the Kriging predictor," Mechanical Systems and Signal Processing, vol. 25, no. 4, pp. 1204-1226, 2011.

[5] J. L. Hu, Q. S. Yan, H. B. Zheng et al., "CFST arch/continuous beam bridge FEM model updating based on Kriging model,"
Journal of Vibration and Shock, vol. 33, no. 14, pp. 33-39, 2014.

[6] H.-F. Lam, J. Hu, and J.-H. Yang, "Bayesian operational modal analysis and Markov chain Monte Carlo-based model updating of a factory building," Engineering Structures, vol. 132, pp. 314-336, 2017.

[7] A. Teughels and G. De Roeck, "Structural damage identification of the highway bridge Z24 by FE model updating," Journal of Sound and Vibration, vol. 278, no. 3, pp. 589-610, 2004.

[8] D. Huang, T. T. Allen, W. I. Notz, and R. A. Miller, "Sequential Kriging optimization using multiple-fidelity evaluations," Structural and Multidisciplinary Optimization, vol. 32, no. 5, pp. 369-382, 2006.

[9] W.-X. Ren and H.-B. Chen, "Finite element model updating in structural dynamics by using the response surface method," Engineering Structures, vol. 32, no. 8, pp. 24552465, 2010.

[10] R. I. Levin and N. A. J. Lieven, "Dynamic finite element model updating using neural networks," Journal of Sound and Vibration, vol. 210, no. 5, pp. 593-607, 1998.

[11] Y. Zhang, Z. C. Hou, and Y. L. Zhao, "Finite element model updating based on response surface of the truncated singular values of frequency response functions," Journal of Vibration Engineering, vol. 30, no. 3, pp. 341-348, 2017.

[12] Z. H. Han, "Kriging surrogate model and its application to design optimization: a review of recent progress," Acta Aeronautica et Astronautica Sinica, vol. 37, no. 11, pp. 3197-3225, 2016.

[13] C. Deng, J. Miao, B. Wei, Y. Feng, and Y. Zhao, "Evaluation of machine tools with position-dependent milling stability based on Kriging model," International Journal of Machine Tools and Manufacture, vol. 124, pp. 33-42, 2018.

[14] J. Vahedi, M. R. Ghasemi, and M. Miri, "An adaptive divergence-based method for structural reliability analysis via multiple Kriging models," Applied Mathematical Modelling, vol. 62, pp. 542-561, 2018.

[15] J. T. Wang, C. J. Wang, and J. P. Zhao, "Frequency response function-based model updating using Kriging model," Mechanical Systems and Signal Processing, vol. 87, pp. 218-228, 2017.

[16] D. D. Zhang and Q. T. Guo, "Application of Kriging response surface in finite element model validation," Journal of $\mathrm{Vi}$ bration and Shock, vol. 32, no. 9, pp. 187-204, 2013.

[17] H. A. Jensen, C. Esse, V. Araya, and C. Papadimitriou, "Implementation of an adaptive meta-model for Bayesian finite element model updating in time domain," Reliability Engineering \& System Safety, vol. 160, pp. 174-190, 2017.

[18] X. Yang, X. Guo, H. Ouyang, and D. Li, “A Kriging model based finite element model updating method for damage detection," Applied Sciences, vol. 7, no. 10, p. 1039, 2017.

[19] Z.-H. Han, Y. Zhang, C.-X. Song, and K.-S. Zhang, "Weighted gradient-enhanced kriging for high-dimensional surrogate modeling and design optimization," AIAA Journal, vol. 55, no. 12, pp. 4330-4346, 2017.

[20] L. Leifsson, S. Koziel, and Y. A. Tesfahunegn, "Multiobjective aerodynamic optimization by variable-fidelity models and response surface surrogates," AIAA Journal, vol. 54, no. 2, pp. 531-541, 2016.

[21] J. D. Tesfahunegn and T. W. Simpson, "Use of Kriging models to approximate deterministic computer models," AIAA Journal, vol. 43, no. 4, pp. 853-863, 2005.

[22] W. Hao, W. Shaoping, and M. M. Tomovic, "Modified sequential Kriging optimization for multidisciplinary complex 
product simulation," Chinese Journal of Aeronautics, vol. 23, no. 5, pp. 616-622, 2010.

[23] X. S. Yang and S. Deb, "Cuckoo Search via lévy flights," in Proceedings of the 2009 World Congress on Nature \& Biologically Inspired Computing (NaBIC), pp. 210-214, IEEE, Coimbatore, India, December 2010.

[24] W. Heylen, S. Lammens, and P. Sar, Modal Analysis Theory and Testing, Katholieke Univ Leuven, Department Werktuigkunde Press, Leuven, Belgium, 2nd edition, 1998. 


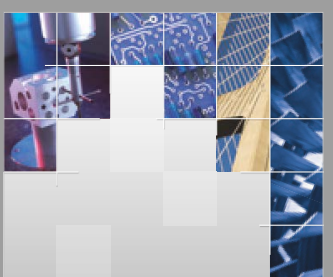

\section{Enfincering}
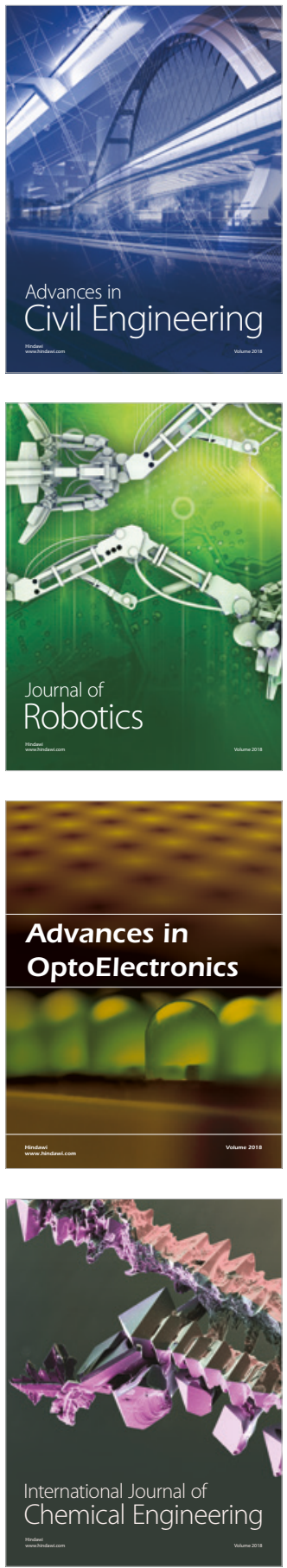

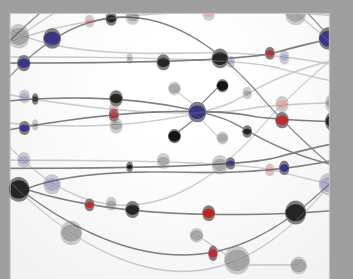

\section{Rotating \\ Machinery}

The Scientific World Journal

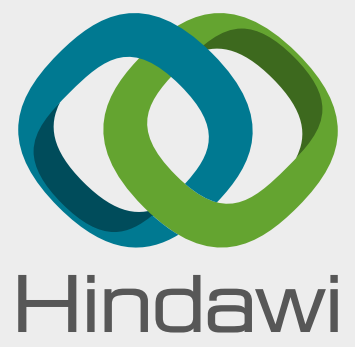

Submit your manuscripts at

www.hindawi.com
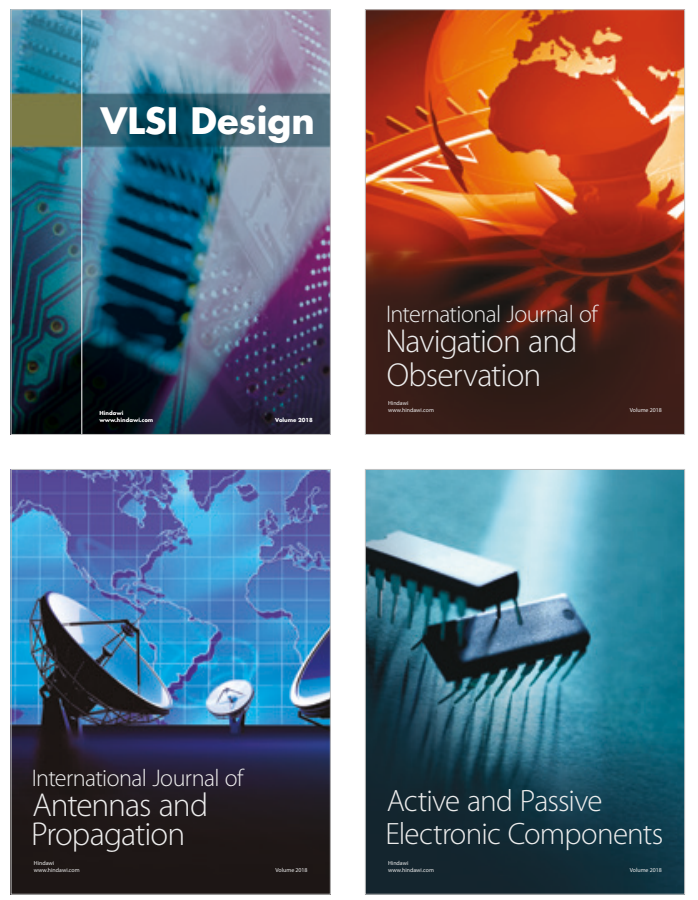
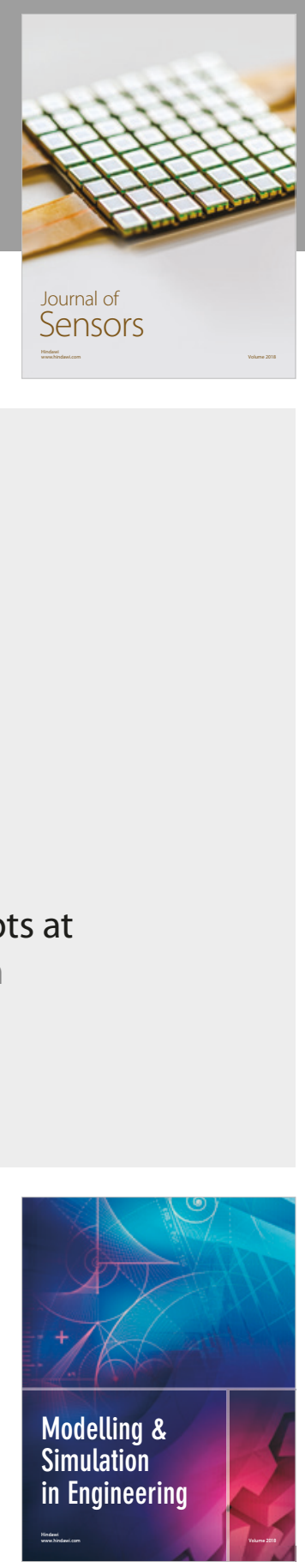

\section{Advances \\ Multimedia}
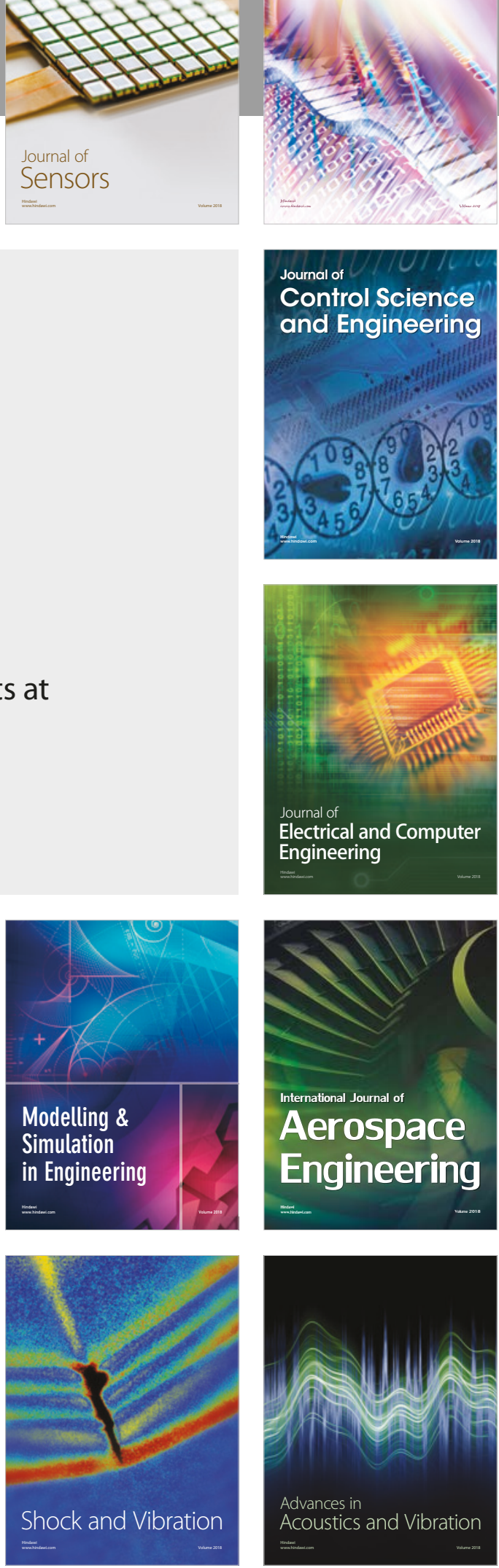